\title{
Efecto de la densidad del tablero y combinación adhesiva sobre propiedades físico-mecánicas críticas en tableros OSB fabricados con maderas nativas chilenas
}

\author{
Effect of board density and adhesive combination on physical and mechanical critical \\ properties of OSB made with Chilean native wood
}

\author{
Rose Marie Garay Moena ${ }^{a *}$, Francisco Damiani Sandoval ${ }^{\text {a }}$ \\ *Autor de correspondencia: ${ }^{\text {a } U n i v e r s i d a d ~ d e ~ C h i l e, ~ F a c u l t a d ~ d e ~ C i e n c i a s ~ F o r e s t a l e s ~ y ~ d e ~ l a ~ C o n s e r v a c i o ́ n ~ d e ~ l a ~ N a t u r a l e z a, ~}$ \\ Departamento de Ingeniería en Maderas y sus Biomateriales, Santa Rosa 11.315, La Pintana, Santiago, Chile, \\ tel.: 56-02-9785724, rgaray@uchile.cl
}

\begin{abstract}
SUMMARY
The aim of this study was to evaluate the effect of board density and adhesive combinations on physic-mechanical properties of industrial scale structural OSB (oriented strand board) made only of strands from Chilean native wood (Nothofagus dombeyi (coigüe), Nothofagus alpina (raulí) and Nothofagus obliqua (roble)). These boards were tested to fulfill the quality standards of the certifier organism "Engineering Wood Association" (APA), since presently they are not being reached. It compared Chilean native wood OSB with common OSB (50\% native and 50\% Pinus radiata wood) as a control. The effect of two densities $\left(700 \mathrm{~kg} \mathrm{~m}^{-3}, 640 \mathrm{~kg} \mathrm{~m}^{-3}\right)$ and four combinations of adhesive (phenol formaldehyde, FF, in the faces and methyl di - isocyanate, MDI, in the core) over the physical and mechanical properties (concentrated static load, dry flexion, cycled humid flexion and linear expansion) was analyzed. The density was the variable with higher incidence on the properties; $700 \mathrm{~kg} \mathrm{~m}^{-3}$ OSB had better physical and mechanical behavior than that presented by $640 \mathrm{~kg} \mathrm{~m}^{-3}$ OSB. We identified an optimal production treatment according to quality standards and physic-mechanical properties. The studied OSB did not show significant differences compared to the control OSB. Finally, it is possible to make certified OSB using Chilean native wood.
\end{abstract}

Key words: OSB, physical and mechanical OSB properties, phenolic adhesives, MDI, adhesive, Chilean native wood.

\section{RESUMEN}

El objetivo de la investigación fue evaluar el efecto de la densidad del tablero y combinación adhesiva sobre algunas propiedades físico-mecánicas de tableros OSB (oriented strand board) estructurales fabricados a escala industrial con hojuelas provenientes de maderas nativas del tipo forestal roble-raulí-coigüe, cuya granulometría se ajustó con el propósito de cumplir con los estándares de calidad exigidos por Engineered Wood Association (APA), organismo certificador de tableros OSB en Chile, ya que actualmente no se están cumpliendo, para esta materia prima. El diseño experimental consistió en fabricar tableros de fibras orientadas (OSB) a nivel industrial y compararlos con tableros OSB testigos (fabricados habitualmente con 50 \% de maderas nativas y 50 \% Pinus radiata. Se analizaron dos densidades de tableros $640 \mathrm{~kg} \mathrm{~m}^{-3}$ y $700 \mathrm{~kg} \mathrm{~m}^{-3}$ y cuatro combinaciones de adhesivo, Fenol Formaldehído (FF) en las caras y metildiisocianato (MDI) en el alma, sobre las propiedades físico-mecánicas: expansión lineal, carga estática concentrada, flexión seca, flexión húmeda ciclada. El factor densidad del tablero, fue la variable con mayor incidencia sobre las propiedades evaluadas, los tableros de $700 \mathrm{~kg} \mathrm{~m}^{-3}$ obtuvo el mejor comportamiento físico-mecánico que los de $640 \mathrm{~kg} \mathrm{~m}^{-3}$. Se identificó un tratamiento óptimo de producción en relación a estándares de calidad y desempeño físico-mecánico. Los tableros de prueba estudiados no mostraron diferencias significativas en relación a los tableros testigos. Se concluye que es factible fabricar tableros estructurales OSB certificados y fabricados con maderas nativas.

Palabras clave: tableros de fibra orientados, OSB, propiedades físicas y mecánicas, adhesivos fenólicos, adhesivos MDI, maderas nativas chilenas.

\section{INTRODUCCIÓN}

Las propiedades físico-mecánicas de los tableros de fibra orientados (oriented strand board = OSB) pueden variar según la materia prima utilizada en su proceso de fabricación. En el caso de la madera de pino radiata (Pinus radiata $\mathrm{D}$. Don), el volumen de madera seca que se necesita por tablero es mayor al de madera nativa, debido a que la densidad de esta última es mayor a la del pino radiata.

En Chile, se utilizan diversos tipos de mezclas de maderas para fabricar tableros OSB según sea la clasificación del mismo, o atendiendo los requerimientos del mercado 
(Louisiana Pacific Chile 2002). Se debe enfrentar a nivel industrial la disyuntiva de abastecimiento de materia prima a base de: lo disponible, los costos y los criterios técnicos para conseguir el equilibrio para una operación industrial altamente eficiente. En los inicios del proceso productivo en Chile, año 2001, se contemplaba producir tableros OSB con $100 \%$ de abastecimiento de maderas nativas de la zona de Panguipulli, región de Los Ríos. Sin embargo, debió incluirse pino radiata, álamo (Populus spp.), eucalipto (Eucalyptus globulus Labill. y Eucalyptus nitens (Dean. et Maiden.) Maiden.), de manera de conseguir por medio de mezclas de especies, los estándares de calidad demandados. De acuerdo a la clasificación de tableros estructurales de APA (American Plywood Association), es posible fabricar tableros para distintos niveles de exigencia. En la norma EN 300 se definen cuatro tipos de tableros OSB en función de sus propiedades mecánicas y resistencia a condiciones húmedas, es decir, según las condiciones ambientales del lugar donde se van a utilizar; estos cuatro grados son:

- OSB/1 tableros para uso general y aplicaciones de interior (incluyendo mobiliario).

- OSB/2 tableros estructurales para utilización en ambiente seco.

- OSB/3 tableros estructurales para utilización en ambiente húmedo.

- OSB/4 tableros estructurales de alta prestación para utilización en ambiente húmedo.

Para dar cumplimiento a estos tipos, se requiere a su vez que las materias primas de origen estén seleccionadas para este objetivo, cosa que hasta ahora no ha sido posible sólo con maderas nativas.

La modificación de la estructura natural de la madera para la transformación a hojuelas, cambia su anisotropía con respeto a sus propiedades originales. La transformación en OSB es más compleja que en otros tipos de tableros y variables como la morfología de partículas, el ordenamiento en el tablero, el método de fabricación, el espesor, la especie de madera, el tipo y cantidad de adhesivo, los aditivos, ciclo de prensa, densidad y grado de compactación entre otros, contribuyen a la producción de tableros con propiedades diferentes (Gatchell et al. 2000, Gu et al. 2005, Garay et al. 2009 y Garay 2009).

La densidad del tablero y consecuentemente su peso, varía dependiendo de cada producto. La densidad típica del tablero se sitúa entre $600 \mathrm{~kg} \mathrm{~m}^{-3}$ y $680 \mathrm{~kg} \mathrm{~m}^{-3}$ (Louisiana Pacific Chile 2002).

La densidad de la madera y la compactación de la estera, definen la densidad final del tablero. Al cambiar la densidad de la madera es necesario realizar ajustes en la compactación de la estera si se desea mantener constante la densidad final del tablero. Sin embargo, las propiedades de los tableros producidos con diferentes densidades de madera y grado de compactación serán distintas, aun cuando la densidad del tablero permanezca constante, in- cluso un tablero hecho con madera menos densa y menor densidad final del tablero puede tener mejores propiedades físico mecánicas de otro fabricado con madera de densidad ligeramente más alta, de densidad final mayor (Linville 2000, Lynne 2001).

El aumento del contenido adhesivo es la manera más fácil de mejorar las propiedades del tablero (Zhang et al. 2007) y compensar debilidades en otros parámetros de fabricación, tales como mala calidad superficial y baja densidad, pero el costo se incrementa. El adhesivo representa el 15 a $20 \%$ de los costos de producción (Poblete 2001, Poblete y Vargas 2006, Okino et al. 2004).

El volumen de madera a incorporar en un tablero, para una densidad determinada, varía dependiendo de la densidad de la materia prima (Poblete 2001, Poblete y Vargas 2006). Estos ajustes afectan las propiedades físicas y mecánicas significativamente.

En un estudio realizado por Pecho et al. (2005) se analizó la influencia de la madera juvenil de pino radiata, en particular el ancho de los anillos de crecimiento y el espesor de la pared celular, sobre las propiedades físicas (hinchamiento y expansión lineal) y mecánicas (adhesión interna, módulo de ruptura (MOR) y módulo de elasticidad (MOE) de tableros de hojuelas orientadas OSB. Los resultados mostraron que la proporción de madera juvenil condiciona las propiedades físicas y mecánicas de los tableros. La tracción normal (0,46 MPa para madera juvenil y 0,70 MPa para madera adulta) y el MOE (3.271 MPa para madera juvenil y de 5.159 MPa para madera madura), concluyendo, que las propiedades del tablero disminuyen significativamente con el incremento de la proporción de madera juvenil.

Se ha determinado que, tanto en tableros de partículas como de fibras de densidad media (medium-density fibreboard $=\mathrm{MDF}$ ), los cambios de la densidad del tablero provocan cambios importantes de las propiedades físicas y mecánicas. En general, un aumento de la densidad del tablero produce incrementos de la flexión y tracción, una disminución de la absorción de agua y un aumento en la expansión, tanto lineal como en el espesor (Poblete et al. 2000, Poblete 2001).

Orellana (2006) logró identificar las propiedades físico-mecánicas que presentaban las mayores dificultades en tableros fabricados con madera nativa. Se evaluaron propiedades de tracción, carga estática concentrada, expansión lineal, hinchamiento en espesor, flexión estática, flexión estática húmeda ciclada y desprendimiento de hojuelas. Se probaron tres densidades de tablero y cinco combinaciones adhesivas. Las propiedades que no lograron integrar los patrones de calidad frente a sus respectivas evaluaciones fueron expansión lineal, flexión estática, flexión estática húmeda ciclada y carga estática concentrada. Estas propiedades fueron designadas como "propiedades críticas". Los mejores resultados se obtuvieron en los tratamientos de densidad $700 \mathrm{~kg} \mathrm{~m}^{-3}$, sin embargo, no se logró determinar la combinación adhesiva óptima para cumplir con los estándares de calidad esperados. 
Basados en los resultados de Orellana (2006), se plantea como hipótesis para esta investigación que modificaciones en la granulometría de hojuelas, densidad y combinación adhesiva permiten cumplir con los estándares de calidad exigidos por APA. De modo que el objetivo fue evaluar el efecto de la densidad del tablero y combinación adhesiva sobre algunas propiedades físico-mecánicas de tableros OSB estructurales fabricados con maderas nativas.

\section{MÉTODOS}

Materiales. La madera en trozas (diámetros entre 15 a $35 \mathrm{~cm}$ y largos de 1,2 m) fue adquirida por la empresa Louisiana Pacific a propietarios particulares de bosques en la zona de Panguipulli. La mezcla de especies nativas que se emplea habitualmente en el proceso incluye $50 \%$ de roble (Nothofagus obliqua (Mirb.) Oerst.), $10 \%$ de raulí (Nothofagus alpina (Poepp. et Endl.) Oerst.) y $40 \%$ de coigüe (Nothofagus dombeyi (Mirb.) Oerst.) Las densidades de la madera ingresada a proceso varían según la especie, para pino radiata entre 320 y $380 \mathrm{~kg} \mathrm{~m}^{-3} \mathrm{y}$ para maderas nativas entre 420 y $480 \mathrm{~kg} \mathrm{~m}^{-3}$. Son descortezadas para luego pasar a la etapa de obtención de hojuelas, siendo ésta la materia prima con la que se fabrican los tableros, caracterizándose por no presentar biodeterioro, y estar a contenidos de humedad cercanos al $100 \%$. Los tableros $100 \%$ con especies nativas están bajo estudio, los que contienen $50 \%$ nativos y $50 \%$ pino radiata, de producción habitual en la fábrica, son tomados como testigos para fines de comparación en esta investigación.

Equipos. Para las evaluaciones de las propiedades mecánicas, se utilizó una máquina de ensayo universal marca INSTRON 4466 modelo M22-12410-12(S), capacidad de carga de $1.020,582 \mathrm{~kg}$ (2.250 libras) y una máquina DEMCO Modelo DEM-PTF 034 con carga máxima de $1.133,89 \mathrm{~kg}$ (2.500 libras). Para las propiedades físicas se empleó una estufa Heraeus; taladro y vainas de metal (eyelettes); dial de medición; estanque de vacío; balanza electrónica Sartorius, serie BL 3100, con precisión de 0,1 g; micrómetro Mitutoyo modelo 223 - 125, rango 0-2,54 cm (0-1”), con precisión de 0,0254 mm (0,001”); pie de metro o vernier digital marca Mitutoyo modelo CD -6 " BS con precisión de $0,0254 \mathrm{~mm}(0,001$ ”). Estos equipos pertenecen a la planta de tableros OSB de Louisina Pacific, en la localidad de Pantanillos, Chile.

De acuerdo a los resultados preliminares de Orellana (2006), las propiedades de tableros fabricados con maderas nativas no cumplieron con los estándares mínimos. Dicha investigación, permitió definir los objetivos de esta nueva experiencia, por lo que se seleccionó la mezcla de especies nativas más adecuadas para la fabricación de tableros OSB, se fabricaron tableros de prueba a nivel de industrial para determinar las propiedades físico-mecánicas de los tableros estructurales OSB y, finalmente, se realizó un análisis comparativo con tableros estructurales OSB de fabricación convencional.
Se estudiaron cuatro combinaciones adhesivas y dos densidades de tableros estructurales OSB fabricados con madera nativa, se evaluó expansión lineal, flexión estática, flexión estática húmeda ciclada y carga estática concentrada y se confrontó con estándares de la certificación APAPS2 (2004) para tableros estructurales. Los resultados se compararon con los tableros estructurales OSB (mezcla de $50 \%$ pino radiata y $50 \%$ de maderas nativas), los cuales son fabricados con mayor frecuencia en Chile. No se consideró como variable la granulometría de hojuelas, puesto que ésta fue estudiada y confirmada mediante un estudio paralelo de Reyes (2008), trabajo ejecutado en la misma fábrica, con los mismos procesos productivos, cuyo objetivo fue modificar el ángulo de contracuchillo para obtener mayor cantidad de hojuelas clasificadas dentro del rango aceptable para la fabricación de tableros OSB con diversas mezclas de especies, incluida $100 \%$ nativas, de esta forma se concluyó que el tamaño de las hojuelas y por consiguiente, su ordenación inciden en las propiedades finales del tablero, por lo que, en esta investigación, se consideró la granulometría recomendada por Reyes (2008).

Los estándares de calidad de los tableros se encuentran certificados por APA a través de PS-2 (Product Standard 2 - Performance Standard for wood-based structural use panels) y definidos detalladamente por Quality Management System II (QMS II), el cual se rige por normas ASTM (E-661, D-1037, D-3043) a través de estas evaluaciones se pretende establecer el comportamiento físico-mecánico de tableros estructurales OSB, utilizando solo maderas nativas como materia prima.

Se modificó la densidad del tablero y la combinación adhesiva (cuadro 1), para poder mejorar las propiedades de los tableros que no cumplieron con los requisitos de las normas. El espesor de los tableros fabricados fue de $11,1 \mathrm{~mm}$. Según los parámetros de corte utilizado por Reyes (2008), el ángulo de contracuchillos de la máquina

Cuadro 1. Diseño experimental para la fabricación industrial de tableros OSB con maderas nativas.

Experimental design for the manufacture of OSB with native

wood.

\begin{tabular}{cccc}
\hline $\begin{array}{c}\text { Identificación } \\
\text { por tratamiento }\end{array}$ & $\begin{array}{c}\text { Densidad } \\
{\left[\mathrm{kg} \mathrm{m}^{-3}\right]}\end{array}$ & \multicolumn{2}{c}{ Combinación adhesiva [\%] } \\
\cline { 3 - 4 } & & $\begin{array}{c}\text { Fenol } \\
\text { formaldehído }\end{array}$ & $\begin{array}{c}\text { Metil } \\
\text { diisocianato }\end{array}$ \\
\hline $\mathrm{A}_{1}$ & 4,20 & 2,20 \\
$\mathrm{~A}_{2}$ & 640 & 4,20 & 2,53 \\
$\mathrm{~A}_{3}$ & & 4,62 & 2,20 \\
$\mathrm{~A}_{4}$ & & 4,62 & 2,53 \\
\hline $\mathrm{B}_{1}$ & & 4,20 & 2,20 \\
$\mathrm{~B}_{2}$ & \multirow{2}{*}{700} & 4,20 & 2,53 \\
$\mathrm{~B}_{3}$ & & 4,62 & 2,20 \\
$\mathrm{~B}_{4}$ & & 4,62 & 2,53 \\
\hline
\end{tabular}


hojuelera (Waferizer) utilizado para la obtención de las hojuelas fue de $45^{\circ}-50^{\circ}$.

El diseño experimental para la fabricación industrial de tableros OSB con maderas nativas (formato $1,22 \times 2,44 \mathrm{~m}$ ) consideró dos niveles de densidad de tablero (640 y $700 \mathrm{~kg}$ $\mathrm{m}^{-3} \mathrm{y}$ cuatro combinaciones adhesivas: fenol formaldehído (FF) 4,20 \% y 4,62 \%, y metil diisocianato (MDI) 2,20\% y $2,53 \%$ (cuadro 1 ).

Los tableros para el estudio se fabricaron en la línea de producción de Louisiana Pacific Chile S.A. en Panguipulli, bajo condiciones industriales. Se fabricaron 128 tableros por tratamiento. El prensado fue en caliente en una prensa multiplato (ocho platos). La temperatura de los platos fue de $230^{\circ} \mathrm{C}$ y presiones de $16 \mathrm{~kg} \mathrm{~cm}^{-2}$; formato final de 1,22 x 2,44 m; tiempo de prensado: 125 segundos; razón de formación de 60:40; velocidad de línea: $0,22 \mathrm{~m} \mathrm{~s}^{-1}$ (43,5 pies/ min); espesor: $11,11 \mathrm{~mm}$ (7/16”); cera: 0,8 \% constante para todas las pruebas, tanto para superficie y centro.

Con el fin de establecer una muestra representativa del lote de producción fueron ejecutadas seis prensadas por condición, de las cuales solo cuatro fueron retenidos para la determinación de las propiedades. Al considerar ocho condiciones en total y seis prensadas por condición de tablero más las dos iniciales se obtiene un total de cincuenta cargas. Debido a la capacidad de la prensa se obtuvieron 64 tableros por cada dos prensadas, por lo tanto, se fabricaron 128 tableros por condición, es decir, dos lotes de 64 .

La muestra seleccionada al azar para los ensayos fue de 10 tableros por tratamiento y el número de repeticiones fue de 10. Los tableros fueron identificados y humectados cuatro días después del proceso de fabricación, se les aplicó un compuesto tenso activo para eliminar la tensión superficial producida en el proceso de prensado, actividad incorporada porque los tableros presentaban pandeo al salir de prensa debido a diferencias de humedad interior-exterior. Posteriormente, fueron almacenados bajo condiciones controladas (65\% de humedad relativa y $20{ }^{\circ} \mathrm{C}$ ) por 10 días antes de realizar la extracción de probetas de ensayo.
El estudio evaluó las propiedades físico-mecánicas que se detallan a continuación.

Determinación de momento máximo (MM) seco, módulo de ruptura (MOR) y módulo de elasticidad (MOE). De acuerdo a la norma ASTM D-3043, en donde se señala que las dimensiones de las probetas son de $11,5 \mathrm{~cm} \times 35,6 \mathrm{~cm}$, con luz entre ambos apoyos de 20,36 cm, tanto en dirección paralela como perpendicular a la formación del tablero. La velocidad de carga en el ensayo es de 2,4 kPa por minuto y está especificada en PS2 (2004) (cuadro 2).

Determinación de flexión húmeda ciclada. Realizada de acuerdo a la norma ASTM D-3043, que define las dimensiones de las probetas $11,5 \mathrm{~cm}$ x $35,6 \mathrm{~cm}$, con luz entre ambos apoyos de 20,36 cm y ASTM D 2559 para el acondicionado de las probetas en vacío presión, en donde se señala que, las muestras fueron totalmente sumergidas en agua a $66{ }^{\circ} \mathrm{C}$ dentro de una cámara de vacío-presión, a vacío de $50,8 \mathrm{kPa}$ (15 in $\mathrm{Hg}$ ) realizado a las muestras por $30 \mathrm{mi}-$ nutos. Luego, fue liberado y las muestras permanecieron por 30 minutos más a presión ambiente. Posteriormente, el agua fue drenada y las muestras fueron llevadas a estufa con una temperatura de $83^{\circ} \mathrm{C}$ por un tiempo de 15 horas; finalmente, las probetas fueron retiradas y ensayadas en la máquina de ensayo universal INSTRON (figura 1A).

Ensayo de carga estática concentrada. Realizado de acuerdo a la norma ASTM E-661. La probeta posee una dimensión de 1,22 x 1,22 m lo que permitió evaluar la resistencia mecánica de un tablero estructural de piso o muro en su medio de servicio, la velocidad de carga durante el ensayo fue de $445 \mathrm{~N}$ por $30 \mathrm{~s}$. Las probetas fueron ensayadas en la máquina DEMCO (figura 1B).

Expansión total al vacío de acuerdo a la norma ASTM D-1037. Las probetas de ensayo, cuyas dimensiones fueron de $7,5 \times 30 \mathrm{~cm}$, fueron colocadas en estufa a $103 \pm$ $2{ }^{\circ} \mathrm{C}$ por 24 horas, luego se midió el espesor, el peso y la

Cuadro 2. Especificaciones de cálculo de flexión para módulo de ruptura y módulo de elasticidad. Calculation specifications for bending (MOR and MOE).

\begin{tabular}{|c|c|c|}
\hline Propiedad & Forma de cálculo & Descripción \\
\hline Momento máximo (MM) (kg m) & $3 P L(b)^{-1}$ & $\mathrm{P}=$ carga máxima en $\mathrm{KN}$ \\
\hline Módulo de ruptura perpendicular (MPa) & $3 P L\left(2 b e^{2}\right)^{-1}$ & $\mathrm{~L}=\mathrm{luz} \geq 24 *$ espesor \\
\hline Módulo de ruptura paralelo (MPa) & $3 P L(b)^{-1}(4 e)^{-1}$ & \\
\hline EI (kg m) & $\Delta P \mathrm{~L}^{3}(4 \Delta Y \mathrm{~b})^{-1}$ & $\begin{array}{l}\text { EI = módulo de elasticidad } x \text { momento de inercia (rigidez } \\
\text { a la flexión). } \Delta P=\text { incremento de la carga (Newton) } \\
\text { en la porción lineal de la curva carga v/s deformación } \\
\Delta Y=\text { incremento de la deflexión en la distancia media } \\
(\mathrm{mm}) \text { correspondiente al incremento de la carga } \mathrm{P}\end{array}$ \\
\hline Módulo de elasticidad perpendicular (MPa) & $\mathrm{L}^{3} \Delta P\left(4 \mathrm{be}^{3} \Delta Y\right)^{-1}$ & $\mathrm{~b}=$ ancho de la probeta en mm \\
\hline Módulo de elasticidad paralelo (MPa) & $\Delta P \mathrm{~L}^{3}(4 \Delta Y \mathrm{~b})^{-1}(\mathrm{e})^{-3}$ & $\mathrm{e}=$ espesor de la probeta en $\mathrm{mm}$ \\
\hline
\end{tabular}



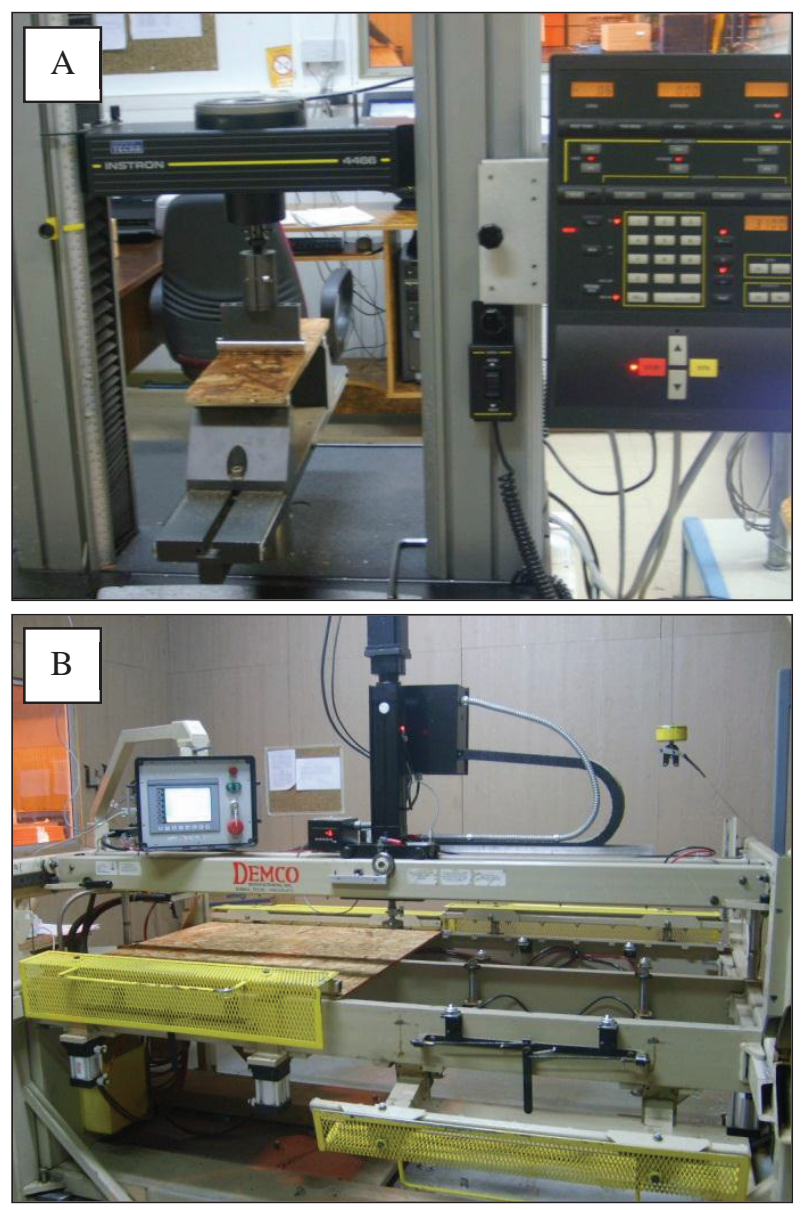

Figura 1. A) Flexión húmeda ciclada, máquina universal Instron. B) Carga estática concentrada, máquina de ensayo DEMCO.

A) Cycled wet bending, Instron universal machine. B) Static load concentrated DEMCO testing machine.

expansión lineal para obtener los valores correspondientes al estado seco. Se colocaron en el estanque al vacío en agua por 1 hora con una presión de $84,7 \mathrm{kPa}(635 \mathrm{~mm} \mathrm{Hg}$ ) y una temperatura de $18 \pm 5^{\circ} \mathrm{C}$, se dejaron por 2 horas más sumergidas en el estanque sin presión, se retiraron y estilaron para su medición. La expansión total se obtiene en porcentaje y fue calculada con la siguiente ecuación:

Expansión lineal $[\%]=\left[\left(\mathrm{L}_{\mathrm{w}}-\mathrm{L}_{\mathrm{d}}\right) 10^{-1}\right] \cdot 100$

Donde, $\mathrm{L}_{\mathrm{w}}=$ dimensión saturada; $\mathrm{L}_{\mathrm{d}}=$ dimensión seca; $10=$ distancia entre eyelettes.

Expansión lineal QMS ${ }^{1}$ II. La prueba fue llevada a cabo en equipos construidos bajo las especificaciones de APA. La expansión lineal fue determinada a partir de probetas con

\footnotetext{
${ }^{1}$ Quality management system. Louisiana Pacific Chile. 2004. Manual de control de calidad (QMS II). Documento interno de la empresa Louisiana Pacific Chile.
}

dimensiones de 3"x12" (76,2 $\mathrm{mm}$ x 304,8 mm). Los eyelettes de bronce estaban ubicados en perforaciones realizadas previamente y limitadas en la línea central a 25,4 mm del extremo. Las muestras se midieron en una plataforma plana y la distancia de las asideras fue medida lo más cercano a 0,0254 mm (0,001”) con una barra tipo compás. Después de la medición las muestras fueron colocadas en forma suelta en una cámara de presión al vacío, completamente sumergidas en agua a $18 \pm 5^{\circ} \mathrm{C}$, y sometidas a un vacío de $84,7 \mathrm{kPa}$ (25 in $\mathrm{Hg}$ ) por una hora.

Posteriormente, el vacío fue liberado y las muestras quedaron sumergidas por dos horas a presión ambiente. Finalmente, fueron retiradas del estanque y se realizó la medición en húmedo. El porcentaje (\%) de expansión lineal fue calculado por la fórmula:

Expansión lineal QMS II $[\%]=\left[\left(\mathrm{L}_{\mathrm{w}}-\mathrm{L}_{\mathrm{i}}\right) 10^{-1}\right] \cdot 100$

Donde, $\mathrm{L}_{\mathrm{w}}=$ dimensión saturada; $\mathrm{L}_{\mathrm{i}}=$ dimensión inicial; $10=$ distancia entre eyelettes.

El modelo estadístico considerado fue

$$
\mathrm{Y}_{\mathrm{ijk}}=\mathrm{m}+\mathrm{t}_{\mathrm{i}}+\mathrm{b}_{\mathrm{j}}+(\mathrm{tb})_{\mathrm{ij}}+\mathrm{e}_{\mathrm{ijk}}
$$

Donde,

$\mathrm{m}=$ media general.

$\mathrm{t}=$ influencia del factor densidad del tablero sobre la media general.

$\mathrm{b}=$ influencia del factor combinación adhesiva sobre la media general.

$\mathrm{tb}=$ influencia de la interacción densidad del tablero y combinación adhesiva sobre la media general.

$\mathrm{e}=$ error experimental.

Las distintas variables obtenidas fueron sometidas a un análisis de varianza (ANDEVA). El valor de probabilidad alfa establecido para calificar como significativas a las diferencias encontradas entre las fuentes de variación fue de 0,05 , así como sus niveles, medidos a través de la prueba de Duncan, utilizados para discernir en las propiedades evaluadas en este estudio.

La variable i representa las densidades del tablero $(i=1,2)$. Los valores de $\mathrm{j}$ representan las combinaciones adhesivas $(j=1,2,3,4)$. La variable $k$ representa las unidades muestrales $(\mathrm{k}=10)$, es decir, el número de tableros ensayados por cada propiedad físico-mecánica. $\mathrm{e}_{\mathrm{ijk}}$ son variables aleatorias independientes estadísticamente $\mathrm{N}\left(0, \mathrm{~s}^{2}\right)$.

\section{RESULTADOS}

Los diversos ensayos definidos por estándares de calidad, representaron en su conjunto el comportamiento de los tableros. Con el propósito de resumir la información obtenida, se optó por presentar cuadros resúmenes que 
permiten comprender los cambios aportados en esta investigación en relación a las propiedades de los tableros OSB.

Las modificaciones iniciales incorporadas, humectación y modificación de ángulo de contracuchillo no fueron incorporadas como variables estadísticas, ya que fueron probadas en otro estudio que realizó Reyes (2008), el cual arrojó un mejoramiento en la granulometría de las hojuelas, por ende, un aumento en el rendimiento y en la calidad de los tableros y porque afecta directamente la habilidad de orientación, implicancia directa en propiedades de flexión.

El análisis estadístico realizado permitió discernir entre tratamientos que pueden ser considerados para dar cumplimiento a la normativa existente, ambos factores estudiados explican los resultados obtenidos en los distintos ensayos, más evidente en la densidad del tablero que en la combinación adhesiva, pues éste no reveló significancia estadística en algunas de las pruebas (deflexión, carga máxima). El análisis bifactorial, mostró que la interacción de factores "densidad de tablero" y "combinación adhesiva" fue estadísticamente significativa sobre expansión paralela y perpendicular. El análisis de medias reveló que los tratamientos $A_{1}$ y $B_{1}$ poseen diferencia significativa entre sus pares y entre sí, siendo $\mathrm{A}_{1}$ la condición peor evaluada para el ensayo de expansión paralela. La condición $\mathrm{A}_{1} \mathrm{y}$ $\mathrm{B}_{4}$ no poseen diferencia estadísticamente significativa y se encuentran sobre el límite superior de decisión. La condición $\mathrm{A}_{4}$ y $\mathrm{B}_{1}$ resultaron no tener contrastes estadísticos significativos, pero se encuentran por debajo del límite inferior de decisión, con lo que se logra identificar ambos tratamientos como los de mejor resultado frente a la evaluación de esta propiedad física. Criterios similares a estos dieron origen a los cuadros 3 y 4.

En el cuadro 3 se presentan los tratamientos que no lograron cumplir con los estándares de calidad de APA, que posee criterios específicos con respecto a la certificación de un tablero estructural, los cuales indican que de un
Cuadro 4. Tratamientos que no cumplieron $(*)$ con los patrones de calidad APA.

Treatments that did not meet APA quality standards.

\begin{tabular}{|c|c|c|c|}
\hline \multirow{2}{*}{$\begin{array}{c}\text { Clave de } \\
\text { identificación }\end{array}$} & \multirow{2}{*}{$\begin{array}{c}\text { Densidad } \\
{\left[\mathrm{kg} / \mathrm{m}^{3}\right]}\end{array}$} & \multicolumn{2}{|c|}{ Combinación adhesiva [\%] } \\
\hline & & $\begin{array}{c}\text { Fenol } \\
\text { formaldehído }\end{array}$ & $\begin{array}{c}\text { Metil } \\
\text { diisocianato }\end{array}$ \\
\hline $\mathrm{A}_{1}^{*}$ & \multirow{4}{*}{640} & 4,20 & 2,20 \\
\hline $\mathrm{A}_{2}{ }^{*}$ & & 4,20 & 2,53 \\
\hline $\mathrm{A}_{3}{ }^{*}$ & & 4,62 & 2,20 \\
\hline $\mathrm{A}_{4}$ & & 4,62 & 2,53 \\
\hline $\mathrm{B}_{1}$ & \multirow{4}{*}{700} & 4,20 & 2,20 \\
\hline $\mathrm{B}_{2}$ & & 4,20 & 2,53 \\
\hline $\mathrm{B}_{3}^{*}$ & & 4,62 & 2,20 \\
\hline $\mathrm{B}_{4}$ & & 4,62 & 2,53 \\
\hline
\end{tabular}

lote de producción, el porcentaje de falla de una propiedad debe ser inferior a un $10 \%$. Si es mayor o igual a un $10 \%$, éste es excluido inmediatamente de aprobación.

En el cuadro 4 se destacan los tratamientos que presentaron fallas por encima del porcentaje de falla aceptado por APA. Se observa que para la densidad más baja la mayoría de los tratamientos no alcanza los niveles mínimos de las propiedades evaluadas, sólo ocurre para la combinación adhesiva más alta, lo que refleja el efecto de compensación que entrega un mayor porcentaje de adhesivo frente a una densidad de tablero más alta. Siguiendo el mismo comportamiento, la densidad más alta sólo el tratamiento B3 no alcanzó los niveles mínimos.

En el cuadro 5 se muestra la comparación de propiedades de los tratamientos estudiados y los tableros industriales. Se observa la variación porcentual (\%) para la combinación adhesiva (fenol formaldehído-metil diisocianato) que pasó de $(3,95 \%-1,97 \%)$ a una combinación adhesiva máxima de $(4,62 \%-2,53 \%)$.

Cuadro 3. Falla por tratamiento según propiedades evaluadas en forma deficiente (\%). Treatment failures for properties evaluated as poor (\%).

\begin{tabular}{|c|c|c|c|c|c|c|c|c|}
\hline \multirow{2}{*}{$\begin{array}{c}\text { Clave de } \\
\text { identificación }\end{array}$} & \multicolumn{2}{|c|}{ ASTM E-661 } & \multicolumn{2}{|c|}{ PS2-04 Método D } & \multicolumn{4}{|c|}{ ASTM D-3043 } \\
\hline & Deflexión & Carga máxima & Falla real & Estimada & MM PL & EI PL & MM PP & EI PP \\
\hline $\mathrm{A}_{1}$ & - & - & $10 *$ & 6 & - & $10 *$ & - & - \\
\hline $\mathrm{A}_{2}$ & $10 *$ & - & 5 & 2 & - & - & - & - \\
\hline $\mathrm{A}_{3}$ & $10 *$ & $10^{*}$ & $45^{*}$ & 42 & - & $10 *$ & - & - \\
\hline $\mathrm{A}_{4}$ & - & - & 5 & 3 & - & - & - & - \\
\hline $\mathrm{B}_{1}$ & - & - & - & 4 & - & - & - & - \\
\hline $\mathrm{B}_{2}$ & - & - & - & 0 & - & - & - & - \\
\hline $\mathrm{B}_{3}$ & - & - & $10^{*}$ & 10 & - & - & - & - \\
\hline $\mathrm{B}_{4}$ & - & - & - & 1 & - & - & - & - \\
\hline
\end{tabular}

*Tratamientos que presentaron fallas por encima del porcentaje de falla aceptado por APA.

$\mathrm{MM}$ = momento máximo. $\mathrm{EI}$ = módulo de elasticidad $\mathrm{x}$ momento de inercia (rigidez a la flexión). 


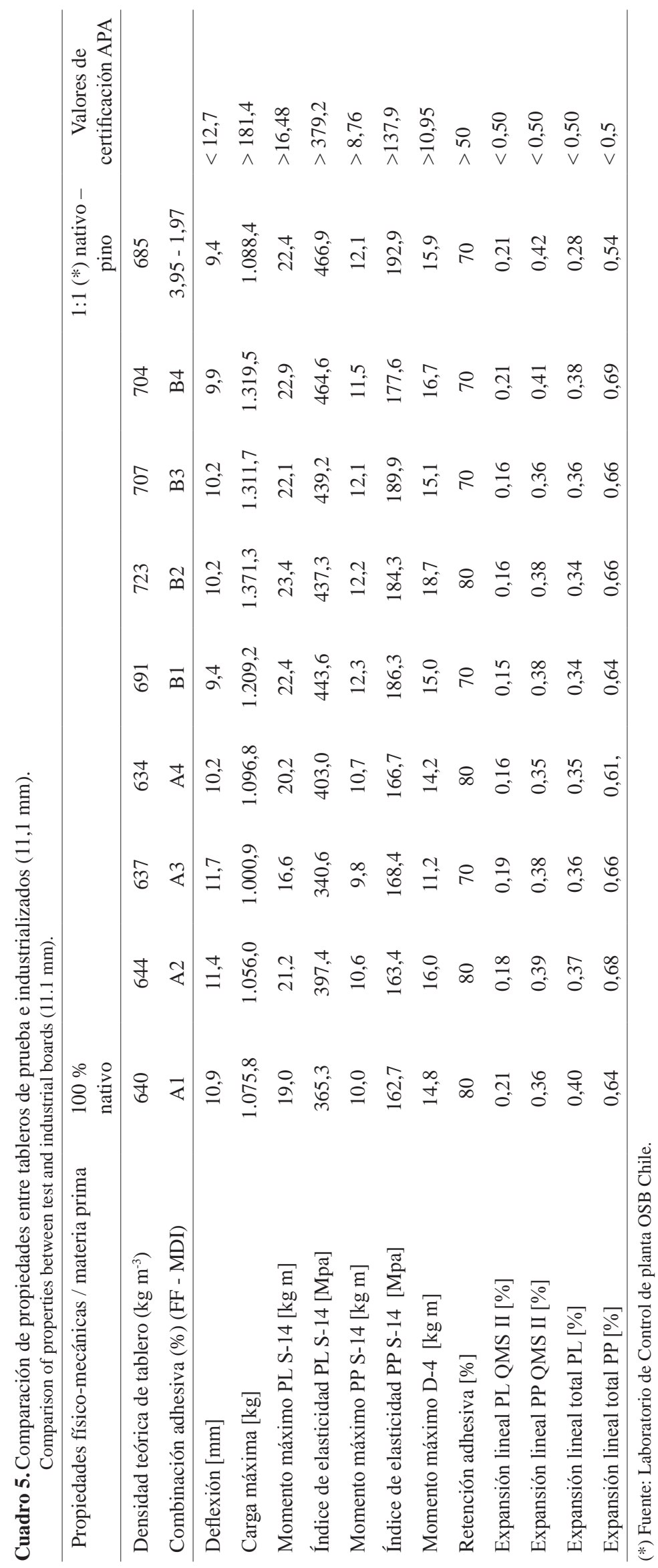




\section{DISCUSIÓN}

Se observa un mejoramiento general de las propiedades físico-mecánicas con la definición de factores específicos, tales como el cambio realizado en el ángulo de contracuchillos del Waferizer y el proceso de humectación que se llevó a cabo cuatro días después del proceso de fabricación de los tableros de prueba. La definición de las variables que se debe considerar como críticas en estudios que incluyen propiedades de tableros OSB han sido informadas ampliamente en la bibliografía internacional y en el país (Gatchel et al. 2000, Okino et al. 2004, Pecho et al. 2005, Poblete y Vargas 2006). Sin embargo, las condiciones particulares para cada proceso productivo deben ser estudiadas bajo la situación específica de cada fábrica y para cada mezcla particular de materia prima y combinación adhesiva. Sólo así es posible definir las propiedades físicas y mecánicas que se quiere obtener de modo de brindar oportunidad para el uso industrial a las maderas nativas para su incorporación en procesos productivos, dada su alta heterogeneidad. Del mismo modo, en relación a las variables de proceso (temperatura, contenido de humedad y tiempos), estas deben ser ajustadas para cada caso en particular, a base de la acumulación de experiencias informadas por diversos investigadores. Debe aclararse que estas variables no fueron incluidas en el análisis, sólo fueron fijadas de acuerdo a la experiencia de condiciones operacionales habituales, las que eventualmente pueden influir en los resultados de manera puntual y debiera evaluarse y aislarse en futuras investigaciones para encontrar un óptimo para cada materia prima y tipo de tablero en proceso; ello, buscando una sincronía fina en el manejo de estas variables que lleve, por ejemplo, a una disminución de la dosis de adhesivo en función de un aumento de la humedad de la manta al ingreso a prensa, con el propósito de hacer más eficiente el proceso por iteración de éstas y otras variables como usualmente ocurre. En este estudio se buscó respuestas respecto a la densidad y a las combinaciones adhesivas, ya que al encontrar combinaciones que sí dan cumplimiento, se produce un impacto en el destino del bosque nativo de la región, pues existen de este modo posibilidades de uso más concretos en este escenario (Linville 2000, Lynne 2001, Gu et al. 2005, Orellana 2006, Sudipto et al. 2006).

La humectación de los tableros permitió reducir la tensión superficial, lo que a su vez provocó estabilidad dimensional y un equilibrio de contenido de humedad sobre los mismos. Esta disminución de tensión tuvo estrecha relación con los resultados obtenidos en los ensayos de expansión lineal paralela y perpendicular, en donde se obtuvo el efecto esperado, es decir, esta propiedad ya no es crítica en el proceso de fabricación de tableros estructurales OSB fabricados con madera nativa. La idea de mejorar la humectación se obtuvo del análisis de los resultados presentados por Geimer y Christiansen (1996), en el cual estudiaron las variables críticas en el curado rápido y la unión de resinas fenólicas.
Según Reyes (2010), la retención de hojuelas en mezclas de alta densidad (nativo, eucalipto/nativo y eucalipto/ pino), tienden a generar mayor cantidad de material fino, en cambio en mezcla de densidad baja (M1), se observa tendencia a generar mayores retenciones de material mediano y material grueso, ello concuerda con que actualmente la mezcla en uso (50 \% nativos y 50\% pino radiata, consigue la granulometría adecuada. Con la modificación incorporada se produjo un aumento en la cantidad de hojuelas aceptadas al emplear $100 \%$ nativas, por lo que es factible incorporarlas a la producción de tableros bajo estándares de APA.

Engineered Wood Association posee criterios específicos con respecto a la certificación de un tablero estructural, los cuales indican que de un lote de producción el porcentaje de falla de una propiedad debe ser inferior a un $10 \%$. Según lo expuesto, si el tratamiento presenta un porcentaje de falla mayor o igual a un $10 \%$, éste es excluido de aprobación, con ello se eliminan las posibilidades de utilizar los factores como variables óptimas de producción al momento de analizar la posibilidad de una futura producción a nivel industrial.

Tomando en cuenta los antecedentes recopilados se puede inferir que la condición óptima de producción sería el tratamiento $\mathrm{A}_{4}\left(640 \mathrm{~kg} \mathrm{~m}^{-3} ; 4,26 \mathrm{FF} / 2,53 \mathrm{MDI}\right)$, esto debido a su desempeño físico-mecánico que es análogo con los exigidos por APA y además de la disminución en los costos de producción al reducir la cantidad de materia prima "madera" por resultar ser el tratamiento de menor densidad de tablero.

La combinación adhesiva utilizada en los tableros fabricados en planta es menor que la de los tratamientos de prueba realizadas en la investigación, lo cual haría pensar que los costos son menores, pero se debe tener en cuenta que el metro ruma de pino posee un costo mayor al de madera nativa. Por consiguiente, los costos se equilibran marcando una tendencia hacia el tratamiento óptimo de producción $\mathrm{A}_{4}$, ya que la densidad de tablero es menor a la densidad habitual de producción en planta, la densidad nominal de los tableros con mezcla nativo-pino fabricados comúnmente en planta es de $685 \mathrm{~kg} \mathrm{~m}^{-3}$ (Louisiana Pacific Chile, 2006).

Aumentando la dosificación de adhesivos y la densidad del tablero se pueden mejorar las propiedades y cumplir con los estándares solicitados, además de mejorar la granulometría de las hojuelas y conseguir adecuadas condiciones de humectación. La consideración más importante está en comprender que estas acciones guardan relación con las necesidades de abastecimiento con materias primas adecuadas para los procesos productivos y que a pesar de que los tableros OSB son fabricados mundialmente para hacer uso de materias primas de menores calidades, tales como residuos de faenas forestales e industriales, la tendencia que prevalece es a que sería preferible conseguir materias primas más homogéneas y estables en su densidad para ser empleadas en estos procesos industriales. 
Este análisis ya ha sido realizado por las empresas, las que saben que es mejor utilizar maderas como álamo $\mathrm{y}$, últimamente, Eucalyptus nitens. Sin embargo, la necesaria relación de equilibrio entre costos y criterios técnicos mantiene siempre en jaque al abastecimiento. Más aún cuando el equilibrio entre necesidad de plantaciones forestales y requerimientos industriales es un tema aún no resuelto en el país, en el cual la tasa de plantación de pino radiata y eucaliptus globulus ha descendido significativamente en los últimos 10 años, pasando de 100.000 ha año ${ }^{-1}$ a sólo 40.000 ha año ${ }^{-1}$. Por otra parte, si no existe uso industrial para la madera nativa, los propietarios de bosques pierden interés en cuidar y mejorar su patrimonio, pues no obtienen rentabilidad, entonces desde este punto de vista, que exista la posibilidad de uso, bajo los planes de manejo establecidos por ley, permite una oportunidad de uso de madera nativa que no es apta para fines más exigentes.

\section{CONCLUSIONES}

La combinación de las variables que fueron modificadas, densidad, combinación adhesiva, ángulo de contracuchillo y humectación permitió mejorar las propiedades físico - mecánicas críticas y acceder a certificación de los tableros OSB fabricados con maderas nativas.

El factor densidad de tablero es la variable estadística con mayor incidencia sobre las propiedades físico-mecánicas críticas de los tableros estructurales OSB fabricados con madera nativa.

El tratamiento $\mathrm{B}_{2}$ es el de mejor desempeño físico-mecánico al evaluar las propiedades críticas de los tableros estructurales OSB fabricados para este estudio.

El tratamiento $\mathrm{A}_{4}$ resulta ser la condición óptima al relacionar estándares de calidad con el desempeño físicomecánico y la reducción en volumen de materia prima (madera).

\section{AGRADECIMIENTOS}

Agradecemos a la empresa Louisiana Pacific Chile, Planta Panguipulli, por brindarnos su apoyo en la ejecución experimental de este estudio.

\section{REFERENCIAS}

ASTM (American Society for Testing Materials, US). 1999. Standard test methods for evaluating properties of woodbase fiber and particle panel materials (D-1037). Annual Book of ASTM Standards. Section 4, Construction vol. 0410 Wood. American society for testing and materials. Easton. 666 p.

ASTM (American Society for Testing Materials, US). 2000. Standard test methods for structural panels in flexure (D3043). Annual Book of ASTM Standards. Section 4, Construction vol. 0410 Wood. American society for testing and materials. Easton. 666 p.

ASTM (American Society for Testing Materials, US). 2003.
Standard test methods for direct moisture content measurement of wood and wood-base materials (D-4442). Annual Book of ASTM Standards. Section 4, Construction vol. 0410 Wood. American society for testing and materials. Easton. 666 p.

ASTM (American Society for Testing Materials, US). 1997. Standard test method for performance of wood and woodbase floor and roof sheathing under concentrated static and impact loads (E-661). Annual Book of ASTM Standards. Section 4, Construction vol. 0410 Wood. American society for testing and materials. Easton. $666 \mathrm{p}$.

Gatchell C, B Heebink, F Hefty. 2000. Influence of components variables on properties of particleboard for exterior use. Forest Product Journal 16(4): 46-59.

Garay R, H Poblete, J Karsulovic. 2009a. Evaluation of oriented strand board and plywood subjected to severe relative humidity and temperature conditions. Forest Products Journal 59(3): 84-90.

Garay R. 2009b. Propiedades de tableros estructurales contrachapados y OSB intemperizados durante dos años. AgroCiencia 25(1): 20-32.

Geimer R, A Christiansen. 1996. Critical variables in the rapid cure and bonding of phenolic resins. Forest Products Journal 46(11/12): 67-72.

Gu H, S Wang, T Neimsuwan S. Wang. 2005. Comparison study of commercial OSB flooring products in thickness swell and mechanical performance. Forest Products Journal 55(12): 239-245.

Linville J. 2000. The influence of a horizontal density distribution on moisture related mechanical degradation of oriented Strand composites. Thesis Master of science in civil engineering. Washington State University. 98 p.

Lynne K. 2001. Impact of strand geometry and orientation on mechanical properties of strand composites. Thesis Master of science in civil engineering. Washington State University. $102 \mathrm{p}$.

Louisiana Pacific Chile. 2002. Evaluación de impacto ambiental. Proyecto de Reconstrucción y modificación de tableros Panguipulli Lousiana Pacific Chile S.A.

Louisiana Pacific Chile. 2006. LP OSB Standard. Disponible en: http://www.lpchile.cl. Consultado 25 sep. 2006.

Okino E, D Teixeira, M de Souza, A Santana, M de Sousa. 2004. Properties of oriented strandboard made of wood species from Brazilian planted forests: Part 1: $80 \mathrm{~mm}-$ long strands of Pinus taeda L. Holz Roh Werkst 62:2 21-224.

Orellana L. 2006. Efecto de la densidad y la cantidad de adhesivo sobre propiedades de tableros OSB. Tesis Ingeniero en Maderas. Valdivia, Chile. Universidad Austral. Facultad de Ciencias Forestales. 124 p.

Pecho R, R Ananias, A Ballerini, A Cloutier. 2005. Influencia de la madera juvenil de pino radiata sobre las propiedades físicas y mecánicas de tableros OSB. Bosque 20(1): 123 - 132.

Poblete H, E Loncomilla, L Inzunza. 2000. Densidad del tablero de partículas y estabilidad dimensional. Bosque 21(2): 69-83.

Poblete H. 2001. Tablero de partículas. Valdivia. Chile. Editorial El Kultrún volumen 1.

Poblete H, R Vargas. 2006. Relación entre densidad y propiedades de tableros HDF producidos por un proceso seco. $\mathrm{Ma}$ dera, Ciencia y tecnología 8(3): 169 - 182.

PS2. 2004. Performance Standard for wood- based structural use panels. Published by APA the Engineered Wood Asso- 
ciation. 56 p.

Reyes C. 2008. Efecto del ángulo de contra cuchillo y mezcla de especies en la clasificación de hojuelas generadas para tableros OSB. Memoria Ingeniero de la Madera. Universidad de Chile. 44 p.

Sudipto D, M Malmbergb, C Frazier. 2007. Cure chemistry of wood/polymeric isocyanate (PMDI) bonds: Effect of wood species. International Journal of Adhesion \& Adhesives 27: $250-257$.

Zhang Y, J Juwan, S Wang. 2007. Effects of resin and wax on the water uptake behavior of wood strands. Wood and Fiber Science 39(2): $271-278$.

Recibido: 18.04 .12

Aceptado: 31.07.12 